\title{
BI-LIPSCHITZ GEOMETRY OF COMPLEX SURFACE SINGULARITIES
}

\author{
LEV BIRBRAIR, ALEXANDRE FERNANDES, AND WALTER D. NEUMANN
}

\begin{abstract}
We discuss the bi-Lipschitz geometry of an isolated singular point of a complex surface which particular emphasis on when it is metrically conical.
\end{abstract}

\section{INTRODUCTION}

A very basic question in metric geometry is whether a neighborhood of a point in an algebraic or semialgebraic set $V$ is metrically conical, i.e., bi-Lipschitz equivalent to a metric cone (our metric is always the "inner metric," given distance within $V$, rather than "outer metric," given by distance in the ambient affine space).

For real algebraic sets an extensive literature exists on local biLipschitz geometry, and failure of metric conicalness is common. The characteristic example is the $\beta$-horn for $\beta=\frac{p}{q} \geq 1$,

$$
\left\{(x, y, z) \in \mathbb{R}^{3}:\left(x^{2}+y^{2}\right)^{q}=z^{2 p}, z \geq 0\right\},
$$

which is topologically the cone on a circle but is bi-Lipschitz classified by $\beta$ [1] and is thus not metrically conical if $\beta>1$. The full biLipschitz classification of germs semi-algebraic sets in this dimension was completed in [1] and [4].

However, the techniques that have been used to distinguish local biLipschitz geometry in the real algebraic case are mostly useless in the complex case. For example, the $\beta$-horns can be distinguished by their volume growth number, defined as

$$
\mu(V, p):=\sup \left\{r>0: \lim _{\epsilon \rightarrow 0} \frac{\operatorname{Vol}\left(V \cap B_{\epsilon}(p)\right)}{\epsilon^{r}}=0\right\} .
$$

This is a well defined rational number for any semialgebraic germ $(V, p)$ (Lion-Rolin [12]), and is bi-Lipschitz invariant (Birbrair and Brasselet [2]), and equals $\beta+1$ for the $\beta$-horn. On the other hand, a complex variety of dimension $n$ has volume growth number equal to $2 n$ at every point. And, in fact, complex algebraic curves are metrically conical at every point.

Key words and phrases. bi-Lipschitz, complex surface singularity. 
It is worth stressing that the local geometry is a bi-Lipschitz invariant of a complex analytic germ $(V, p)$ (independent of embedding). For if one uses any set of generators of the local ring $\mathcal{O}_{p}(V)$ to embed $(V, p)$ in some $\left(\mathbb{C}^{N}, 0\right)$, then $(V, p)$ inherits a Riemannian metric at smooth points of $V$ which gives a distance metric on $(V, p)$ that is unchanged up to bi-Lipschitz equivalence when adding to the set of generators of $\mathcal{O}_{p}(V)$ that is used.

The first examples of failure of metric conicalness in the complex setting were given by the first two authors in [5]. They demonstrated that the $A_{k}$-singularity $z^{k+1}=x^{2}+y^{2}$ is not metrically conical for $k$ odd and $\geq 3$. $A_{k}$ is weighted homogeneous with weights $\left(\frac{k+1}{2}, \frac{k+1}{2}, 1\right)$. The current authors showed much more generally:

Theorem 1.1 ([6]). A weighted homogeneous surface singularity is not metrically conical if its two lowest weights are distinct.

A singularity is homogeneous if it is weighted homogeneous with all its weights equal. Homogeneous singularities are (obviously) metrically conical; the converse holds for cyclic quotient singularities:

Theorem $1.2\left([6)\right.$. A cyclic quotient singularity $\mathbb{C}^{2} /(\mathbb{Z} / n)$ is metrically conical if and only if it is homogeneous.

Since the two lowest weights are equal to each other for many nonhomogeneous cyclic quotient singularities, the converse of Theorem 1.1 is not necessarily true. But we will prove here a converse to Theorem 1.1 for Brieskorn hypersurfaces:

Theorem 1.3. The Brieskorn singularity

$$
V(a, b, b):=\left\{(x, y, z) \in \mathbb{C}^{3}: \alpha_{0} x^{a}+\alpha_{1} y^{b}+\alpha_{2} z^{b}=0\right\}
$$

with $a<b$ has a metrically conical singularity at 0 for any $\alpha_{0}, \alpha_{1}, \alpha_{2} \in$ $\mathbb{C}-\{0\}$.

We close this introduction by sketching the known obstructions to metric conicalness in the complex case.

Let $M_{\epsilon}$ be the link of the point $p \in V$ (the boundary of $V \cap B_{\epsilon}(p)$, where $B_{\epsilon}(p)$ is an $\epsilon$-ball in some ambient affine space in which $(V, p)$ is embedded, $\epsilon$ sufficiently small). A non-trivial homology class or free homotopy class in $M_{\epsilon}$ will have a lower bound on the diameter of any cycle representing it in $M_{\epsilon}$. In a metric cone this lower bound will shrink at most linearly with respect to $\epsilon$ as $\epsilon \rightarrow 0$. If such a cycle exists which shrinks faster than linearly, it therefore obstructs metric conicalness. Theorems 1.1 and 1.2 are proved by exhibiting such fast cycles for $\pi_{1}(M)$. This is close to the ideas of metric homology [2, 3], 
but the link of a surface singularity may be a homology sphere, in which case homology is not useful. Instead, one might see this as a first step to "metric homotopy theory" in bi-Lipschitz geometry.

A main tool used in [5] was to exhibit a "Cheeger cycle" in $V$ (also called "separating set"), a codimension 1 subset that divides $V$ into pieces of roughly equal volume, but whose 3-dimensional volume shrinks faster towards $p$ than it could in a metric cone. In [5] the Cheeger cycle was constructed as the union of orbits of the real points of $V$ under the $\mathbb{C}^{*}$-action, so it was important that an appropriate real form be used. At the time it was also only known that this Cheeger cycle obstructs $(V, p)$ being semi-algebraicly bi-Lipschitz equivalent to a metric cone. We are grateful to Bruce Kleiner for showing us how the semi-algebraic condition on the bi-Lipschitz equivalence can be removed.

In the final section of this paper we revisit the separating set approach, describing a more robust version using "conflict sets." We then sketch how it can be used to show that the Briançon-Speder family of singularities of constant topological type does not have constant bi-Lipschitz type. The details of this argument will appear in a future paper [7]. The embedded real case of this, which is much more elementary, was proved in [11.

Acknowledgements. The authors acknowledge support for this research under the following grants: CNPq grant no 300985/93-2 (Birbrair), CNPq grant no 300393/2005-9 (Fernandes), NSA grant H9823006-1-011 and NSF grant no. DMS-0206464 (Neumann). Birbrair and Neumann express their gratitude to the ICTP in Trieste for its hospitality during the final work on this paper.

\section{Conical Brieskorn singularities}

This section is devoted to the proof of Theorem 1.3; we refer to the notation of that theorem. Since changing the coefficients $\alpha_{i}$ can be realized by a linear change of coordinates in $\mathbb{C}^{3}$, which is bi-Lipschitz, we can choose the coefficients at our convenience. We choose

$$
V=\left\{(x, y, z) \in \mathbb{C}^{3}: x^{a}+y^{b}-z^{b}=0\right\} .
$$

Then the projection of $V$ to the $(y, z)$-plane is an $a$-fold branched cover branched along the lines $z=\omega y$ with $\omega \in \mu_{b}$, the $b$-th root of unity. We will show that this projection has a bounded Lipschitz constant except in a thin neighborhood of the branch locus. The conical structure of the $(y, z)$-plane pulls back to $V$ except in these thin neighborhoods. 
We then show that these neighborhoods can be chosen to also carry a conical structure.

We first decompose $V$ into pieces. When we refer to $V$ and its pieces we will really mean the germ at 0 , so $V$ is always implicitly intersected with a small neighborhood of $0 \in \mathbb{C}^{3}$.

Our two pieces will consist of a disk-bundle neighborhood of the branch set of the projection to the $(y, z)$-plane and the closure of its complement, and we will show that both pieces are metrically conical at 0 . But we start with a preliminary decomposition into two pieces which are not metrically conical.

Our preliminary decomposition of $V$ is as follows:

$$
\begin{aligned}
& V_{0}:=\left\{(x, y, z) \in V:|x|^{2 a-2} \geq|y|^{2 b-2}+|z|^{2 b-2}\right\} \\
& V_{1}:=\left\{(x, y, z) \in V:|x|^{2 a-2} \leq|y|^{2 b-2}+|z|^{2 b-2}\right\} .
\end{aligned}
$$

Using the fact that $x^{a}=-\left(y^{b}-z^{b}\right)$ on $V$, we can write this as

$$
\begin{aligned}
& V_{0}:=\left\{(x, y, z) \in V:\left|y^{b}-z^{b}\right|^{(2-2 / a)} \geq|y|^{2 b-2}+|z|^{2 b-2}\right\} \\
& V_{1}:=\left\{(x, y, z) \in V:\left|y^{b}-z^{b}\right|^{(2-2 / a)} \leq|y|^{2 b-2}+|z|^{2 b-2}\right\} .
\end{aligned}
$$

Denote the images of the projections to the $(y, z)$-plane by

$$
\begin{aligned}
& W_{0}:=\left\{(y, z) \in \mathbb{C}^{2}:\left|y^{b}-z^{b}\right|^{(2-2 / a)} \geq|y|^{2 b-2}+|z|^{2 b-2}\right\} \\
& W_{1}:=\left\{(y, z) \in \mathbb{C}^{2}:\left|y^{b}-z^{b}\right|^{(2-2 / a)} \leq|y|^{2 b-2}+|z|^{2 b-2}\right\} .
\end{aligned}
$$

Lemma 2.1. The projection of $V$ to the $(y, z)$-plane is an a-fold cyclic covering branched along the lines $z=\omega y$ with $\omega \in \mu_{a}$ (the a-th roots of unity). When restricted to $V_{0}-\{0\}$ it is a bi-Lipschitz unramified covering of its image $W_{0}-\{0\}$ with Lipschitz constant $\sqrt{1+\frac{b^{2}}{a^{2}}}$.

Proof. Write $f(x, y, z)=x^{a}+y^{b}-z^{b}$ so $V=f^{-1}(0)$. That $V \rightarrow \mathbb{C}^{2}$ is a cyclic branched cover follows because it is the orbit map of $\mu_{a}$ acting on $V$ by multiplication in the $x$-coordinate. Branching is thus along $x=0$ which projects to the set $y^{b}=z^{b}$ in $\mathbb{C}^{2}$. This is the set $\left\{(y, z): z=\omega y, \omega \in \mu_{a}\right\}$. The restriction to $V_{0}$ is an unramified cover since $W_{0}-\{0\}$ does not intersect this branch locus.

The bi-Lipschitz constant of the projection at a point of $V$ will be the bi-Lipschitz constant of the projection of the tangent plane at that point to the $(y, z)$-plane. The tangent plane at the point is given by the orthogonal complement of the complex gradient $\overline{\nabla f}$. The following lemma is an exercise:

Lemma 2.2. For planes complex orthogonal to unit vectors $u_{1}$ and $u_{2}$ the orthogonal projection of one plane to the other has bi-Lipschitz constant $1 /\left|\left\langle u_{1}, u_{2}\right\rangle\right|$, where $\langle$,$\rangle is hermitian inner product.$ 
Returning to the proof of Lemma 2.1, the unit vectors in question are

$$
\frac{\overline{\nabla f}}{|\nabla f|}=\frac{\left(a \bar{x}^{a-1}, b \bar{y}^{b-1},-b \bar{z}^{b-1}\right)}{\sqrt{a^{2}\left|x^{a-1}\right|^{2}+b^{2}\left(\left|y^{b-1}\right|^{2}+\left|z^{b-1}\right|^{2}\right)}}
$$

and $(1,0,0)$. So the bi-Lipschitz constant is

$\frac{\sqrt{a^{2}\left|x^{a-1}\right|^{2}+b^{2}\left(\left|y^{b-1}\right|^{2}+\left|z^{b-1}\right|^{2}\right)}}{\left|a x^{a-1}\right|} \leq \frac{\mid \sqrt{a^{2}\left|x^{a-1}\right|^{2}+b^{2}\left|x^{a-1}\right|^{2}}}{\left|a x^{a-1}\right|}=\sqrt{1+\frac{b^{2}}{a^{2}}}$,

where the inequality uses the defining inequality for $V_{0}$.

Note that $W_{0}-\{0\}$ and $W_{1}-\{0\}$ decompose $\mathbb{C}^{2}-\{0\}$ into two subsets that meet along their boundaries. We claim:

Lemma 2.3. Assume $b>a$. Then, in a neighborhood of $0, W_{1}-\{0\}$ consists of disjoint closed disk-bundle neighbourhoods of the lines $z=$ $\omega y$. At small distance $r$ from the origin these disks have radius close to $\mathrm{cr}^{(b-1) /(a-1)}$ for some $c>0$.

Proof. Since the $\mu_{b}$-action that multiplies just the $z$-coordinate by $\omega$ permutes the lines in question, it suffices to consider the line $y=$ $z$. For fixed $y=v$ a transverse section to this line can be given by $(v(1-\xi), v(1+\xi))$ as $\xi$ varies. We restrict $\xi$ to be small so we are in a neighbourhood of the line and we consider the set of $(v(1-\xi), v(1+\xi)) \in$ $\mathbb{C}^{2}$ satisfying

$$
\left|\left(v^{b}(1-\xi)^{b}-v^{b}(1+\xi)^{b}\right)\right|^{(2-2 / a)} \leq|v(1-\xi)|^{2 b-2}+|v(1+\xi)|^{2 b-2} .
$$

This inequality simplifies to:

$$
\left|(1-\xi)^{b}-(1+\xi)^{b}\right|^{2(a-1) / a} \leq|v|^{2(b-a) / a}\left(|1-\xi|^{2 b-2}+|1+\xi|^{2 b-2}\right)
$$

To first order in $\xi$ this is

$$
|2 b \xi|^{2(a-1) / a} \leq 2|v|^{2(b-a) / a},
$$

which gives

$$
\sqrt{2}|v \xi| \leq c|v|^{(b-1) /(a-1)},
$$

for some constant $c$ (specifically, $c=\sqrt{2} /\left(2^{(a-2) /(2 a-2)} b\right)$ ).

Since the radius of the transverse section at $v$ is the maximum of $\sqrt{2}|v \xi|$, the lemma follows.

Denote the branch locus $y^{b}=z^{b}$ in $V$ or in the $(y, z)$-plane by $B$ (we use the same notation for both).

Note that the radius of the disk-bundle neighbourhood $W_{1}$ of $B$ at distance $r$ from the origin is of order $r^{(b-1) /(a-1)}$, which is $o(r)$. Thus 
if we choose a small $\delta>0$ and decompose $V$ conically with respect to the $(y, z)$-plane as follows:

$$
\begin{aligned}
& C_{0}:=\{(x, y, z) \in V: d((y, z), B) \geq \delta|(y, z)|\} \\
& C_{1}:=\{(x, y, z) \in V: d((y, z), B) \leq \delta|(y, z)|\},
\end{aligned}
$$

with images in $\mathbb{C}^{2}$ :

$$
\begin{aligned}
& D_{0}:=\left\{(y, z) \in \mathbb{C}^{2}: d((y, z), B) \geq \delta|(y, z)|\right\} \\
& D_{1}:=\left\{(y, z) \in \mathbb{C}^{2}: d((y, z), B) \leq \delta|(y, z)|\right\},
\end{aligned}
$$

then, so long as we are in a small enough neighborhood of $0 \in V$, the sets $C_{0}$ and $D_{0}$ are subsets of $V_{0}$ and $W_{0}$. Since $D_{0}$ is strictly conical and $C_{0}$ is a bi-Lipschitz covering of it, $C_{0}$ is metrically conical. To complete the proof of the theorem we must just show that the other piece, $C_{1}$, is also metrically conical, since it follows from Corollary 0.2 of 13 that the union is then metrically conical.

$C_{1}$ is a union of disk-bundle neighbourhoods of the lines $y=\omega z$. As before, it suffices to focus just on the component $C_{1}^{\prime}$, which is a neighborhood of $y=z$. The proof of Theorem 1.3 is then completed by the following lemma.

Lemma 2.4. The map of $C_{1}^{\prime}$ to $\mathbb{C}^{2}$ given by

$$
(x, y, z) \mapsto\left(\frac{y+z}{2}, e^{i \arg x} \sqrt{|x|^{2}+\frac{|y-z|^{2}}{4}}\right)
$$

is a bi-Lipschitz homeomorphism onto a metric cone in $\mathbb{C}^{2}$.

The rest of this section is devoted to proving this lemma. We first introduce more convenient coordinates on $C_{1}^{\prime}$. Define

$$
u:=\frac{z-y}{2} \quad v:=\frac{z+y}{2}
$$

The inverse change of coordinates is

$$
y:=v-u \quad z:=v+u
$$

Thus $v$ is as in the proof of Lemma 2.3 and $u$ equals $v \xi$ in the notation of that proof. $C_{1}^{\prime}$ is given by

$$
C_{1}^{\prime}=\left\{(x, u, v) \in \mathbb{C}^{3}: x^{a}=(v+u)^{b}-(v-u)^{b},|u| \leq \delta|v|\right\}
$$

and the map of Lemma 2.4 is

$$
F(x, u, v)=\left(r_{v} e^{i \theta_{v}}, e^{i \theta_{x}} \sqrt{r_{x}^{2}+r_{u}^{2}}\right)
$$

where we are using polar coordinates

$$
x=r_{x} e^{i \theta_{x}}, \quad u=r_{u} e^{i \theta_{u}}, \quad v=r_{v} e^{i \theta_{v}} .
$$


Since for $v$ small the size of $x$ is negligible with respect to $\delta|v|$, the image of $F$ is extremely close to the conical set $\{(u, v):|u| \leq \delta v\}$, and the main issue is checking the bi-Lipschitz bound.

We can rewrite the defining equation $x^{a}=(v+u)^{b}-(v-u)^{b}$ in (2) as

$$
x^{a}=2 b u v^{b-1}+u^{3} g(u, v)
$$

with $g(u, v)$ a polynomial of degree $b-3$. If we choose $\delta$ very small in (2) then $u^{3} g(u, v)$ will be virtually negligible, so to simplify calculation we will omit this term for now and work with

$$
C:=\left\{(x, u, v) \in \mathbb{C}^{3}: x^{a}=2 b u v^{b-1},|u| \leq \delta|v|\right\}
$$

instead of $C_{1}^{\prime}$. It is fairly clear that up to bi-Lipschitz equivalence this changes nothing, but we come back to this issue later.

It is helpful to first consider a single transverse section to the $v$-line, so, writing $D=1 /\left(2 b v^{b-1}\right)$, we start by proving

Lemma 2.5. For any integer $a \geq 1$ and any $D \in \mathbb{C}^{*}$ the map $f$ of the graph $S_{D}:=\left\{(x, u): u=D x^{a}\right\}$ to $\mathbb{C}$ given by

$$
f(x, u)=e^{i \theta_{x}} \sqrt{r_{x}^{2}+r_{u}^{2}}=e^{i \theta_{x}} \sqrt{r_{x}^{2}+|D|^{2} r_{x}^{2 a}},
$$

is a bi-Lipschitz homeomorphism with bi-Lipschitz bound $\leq a$.

Proof. The metric in $S_{D}$ is given by

$$
\begin{aligned}
d s^{2} & =|d x|^{2}+|d u|^{2}=|d x|^{2}+\left|a D x^{a-1}\right|^{2}|d x|^{2} \\
& =\left(d r_{x}{ }^{2}+r_{x}^{2} d \theta_{x}^{2}\right)\left(1+a^{2}|D|^{2} r_{x}^{2 a-2}\right) .
\end{aligned}
$$

On the other hand, differentiating $f$ gives

$$
d f=e^{i \theta_{x}}\left(\frac{r_{x}+a|D|^{2} r_{x}^{2 a-1}}{\sqrt{r_{x}^{2}+|D|^{2} r_{x}^{2 a}}} d r_{x}+i \sqrt{r_{x}^{2}+|D|^{2} r_{x}^{2 a}} d \theta_{x}\right),
$$

so the metric pulled back by $f$ is

$$
|d f|^{2}=\frac{\left(1+a|D|^{2} r_{x}^{2 a-2}\right)^{2}}{1+|D|^{2} r_{x}^{2 a-2}} d r_{x}^{2}+\left(1+|D|^{2} r_{x}^{2 a-2}\right) r_{x}^{2} d \theta_{x}^{2}
$$

The ratio of coefficients of $r_{x}^{2} d \theta_{x}^{2}$ in (7) and (9) increases steadily from 1 to $a^{2}$ as $r_{x}$ increases from 0 to $\infty$. And it is an exercise to check that the ratio of the coefficient of $d r_{x}{ }^{2}$ increases from 1 to $\frac{(a+1)^{2}}{4 a}$ and then decreases again to 1 as $|D|^{2} r_{x}^{2 a-2}$ goes from 0 to $\infty$ via $1 / a$. Since $\frac{1}{a^{2}} \leq \frac{(a+1)^{2}}{4 a} \leq a^{2}$ for all $a \geq 1$, it follows that the ratio $d s^{2} /|d f|^{2}$ is bounded below and above by $\frac{1}{a^{2}}$ and $a^{2}$, so the bi-Lipschitz constant of $f$ is bounded by $a$. 
Consider now a point $\left(x_{0}, u_{0}, v_{0}\right) \in C$. At this point we have two surfaces: the surface $C=\left\{x^{a}=2 b u v^{b-1}\right\}$ that we are interested in, and the surface

$$
C_{\left(x_{0}, u_{0}, v_{0}\right)}:=\left\{(x, u, v): x^{a}=2 b u v_{0}^{b-1}\right\}
$$

which is the product of the $v$-plane with the curve of Lemma 2.5. For each of these surfaces we can consider the local bi-Lipschitz constant of the map $F(x, u, v)=\left(v, e^{i \theta_{x}} \sqrt{r_{x}^{2}+r_{u}^{2}}\right)$. For $C_{\left(x_{0}, u_{0}, v_{0}\right)}$ we have already computed this and it is uniformly bounded by $a$. The constants for $C$ and $C_{\left(x_{0}, u_{0}, v_{0}\right)}$ have ratio bounded by the bi-Lipschitz constant of the projection between the tangent spaces of these two surfaces at the given point. So it remains to compute this latter number and show it is uniformly bounded.

The gradients of the two surfaces at the given point are

$$
\left(a x_{0}^{a-1}, 2 b v_{0}^{b-1}, 2 b(b-1) u_{0} v_{0}^{b-2}\right) \quad \text { and } \quad\left(a x_{0}^{a-1}, 2 b v_{0}^{b-1}, 0\right)
$$

respectively. Referring to Lemma 2.3 we see that the number in question is

$$
\frac{\sqrt{\left|a x_{0}^{a-1}\right|^{2}+\left|2 b v_{0}^{b-1}\right|^{2}+\left|2 b(b-1) u_{0} v_{0}^{b-2}\right|^{2}}}{\sqrt{\left|a x_{0}^{a-1}\right|^{2}+\left|2 b v_{0}^{b-1}\right|^{2}}}
$$

Note that the additional term in the numerator is at most $((b-1) \delta)^{2}$ times the term preceding it, so the whole expression in (10) is bounded by $\sqrt{1+((b-1) \delta)^{2}}$. This completes the proof of this point.

Finally, we promised to revisit the issue of replacing $C_{1}^{\prime}$ by $C$ at equation (5). This is a similar argument to the one we have just completed - one checks that the projection in the $x$-direction between $C$ and $C_{1}^{\prime}$ has bi-Lipschitz constant bounded by $\left(1+\delta^{2} g(\delta)\right)$ for a certain fixed degree $b-3$ polynomial in $\delta$, and can hence be made arbitrarily close to 1 by choosing $\delta$ small enough.

\section{Separating sets and the BrianÇOn-Speder family}

For an algebraic germ $(X, p)$, the $r$-density of $X$ at $p$ is defined as

$$
\operatorname{density~}_{r}(X, p)=\lim _{\epsilon \rightarrow 0} \frac{\operatorname{vol}\left(X \cap B_{\epsilon}(p)\right)}{\epsilon^{r}}
$$

Thus, the volume growth number, defined in the Introduction, is

$$
\mu(X, p)=\sup \left\{r>0: \operatorname{density}_{r}(X, p)=0\right\} .
$$

Although the value of the $r$-density is not generally a bi-Lipschitz invariant, its vanishing or non-vanishing is, which is why $\mu$ also is a bi-Lipschitz invariant. If $r=\operatorname{dim} X$ we speak simply of the density. 
Let $(X, p)$ be an $n$-dimensional germ and $(Y, p) \subset(X, p)$. Then $(Y, p)$ is called a separating set if

- $Y$ divides $X$ into two pieces $X_{1}$ and $X_{2}$, each containing $p$;

- $\operatorname{density}_{n-1}(Y)=0, \operatorname{density}_{n}\left(X_{1}\right) \neq 0$, $\operatorname{density}_{n}\left(X_{2}\right) \neq 0$.

In view of the comments above, the existence of a separating set is a bi-Lipschitz invariant. It is also an obstruction to metric conicalness:

Proposition 3.1. A metric cone cannot contain a separating set.

With extra conditions on semialgebraicity of the conical structure and sets in question this is in [5]. Bruce Kleiner showed us how to eliminate the extra assumptions; details will appear in [7].

In 55 a separating set was used to show the non-conicalness of the $A_{k}$ singularity for odd $k \geq 3$. We here give a different construction that works for all $k \geq 2$. Note that these are also covered by Theorem 1.1, where the proof is by fast cycles; we describe the separating set approach to illustrate its usefulness.

Consider therefore $A_{k}$, written in the form $V=\left\{(x, y, z): z^{k+1}=\right.$ $x y\}$. The subset $\{z=0\} \cap V$ has components $L_{1}=\{x=0\} \cap V$ and $L_{2}=\{y=0\} \cap V$. Consider their conflict set

$$
Y=\left\{p \in V: d\left(p, L_{1}\right)=d\left(p, L_{2}\right),\right.
$$

where $d()$ is distance. A symmetry argument shows that $Y$ separates $V$ into two isometric pieces $V_{1}$ and $V_{2}$, so

$$
\operatorname{density}_{4}\left(V_{1}\right)=\operatorname{density}_{4}\left(V_{2}\right)=\frac{1}{2} \operatorname{density}_{4}(V) \neq 0 .
$$

Any smooth arc on $Y$ that approaches 0 has $|x|=|y|=|z|^{(k+1) / 2}$ and must therefore approach tangent to the $z$-axis, so the tangent cone of $Y$ is the $z$-axis. However, if a semialgebraic set $Y$ of dimension $n$ has tangent cone of lower dimension, then $\operatorname{density}_{n}(Y)=0$ (see Federer [10]). Thus $Y$ is a separating set.

The Briançon-Speder family 9]

$$
V_{t}=\left\{(x, y, z): z^{15}+z y^{7}+x^{5}+t x y^{6}=0\right\}
$$

is a family of weighted homogeneous surface singularities with weights $(3,2,1)$ which is topologically a trivial family but which does not satisfy the Whitney conditions. By Theorem 1.1, no $V_{t}$ is metrically conical.

Theorem 3.2. The bi-Lipschitz type is non-constant in the BriançonSpeder family: $V_{t}$ has a separating set for $t \neq 0$ and none for $t=0$.

As already mentioned, details will be in [7]. 
10 LEV BIRBRAIR, ALEXANDRE FERNANDES, AND WALTER D. NEUMANN

\section{REFERENCES}

[1] Lev Birbrair Local bi-Lipschitz classification of 2-dimensional semialgebraic sets. Houston J. Math. 25 (1999), 453-472.

[2] Lev Birbrair, Jean-Paul Brasselet, Metric homology. Comm. Pure Appl. Math. 53 (2000), 1434-1447.

[3] Lev Birbrair, Jean-Paul Brasselet, Metric homology for isolated conical singularities. Bull. Sci. Math. 126 (2002), 87-95.

[4] Lev Birbrair, Alexandre C. G. Fernandes, Metric theory of semialgebraic curves. Rev. Mat. Complut. 13 (2000), 369-382.

[5] Lev Birbrair and Alexandre Fernandes, Metric geometry of complex algebraic surfaces with isolated singularities, Comm. Pure Appl. Math. (to appear).

[6] Lev Birbrair, Alexandre Fernandes, and Walter D. Neumann, Bi-Lipschitz geometry of weighted homogeneous surface singularities, Math. Ann. (to appear).

[7] Lev Birbrair, Alexandre Fernandes, and Walter D. Neumann, $\mu$-constant does not imply bi-Lipschitz triviality, in preparation

[8] JP Brasselet, M Goresky, R MacPherson, Simplicial differential forms with poles. Amer. J. Math. 113 (1991), 1019-1052.

[9] Joël Briançon and Jean-Paul Speder, La trivialité topologique n'implique pas les conditions de Whitney. C. R. Acad. Sci. Paris Sér. A-B 280 (1975), A365-A367.

[10] Herbert Federer, Geometric measure theory. Die Grundlehren der mathematischen Wissenschaften, Band 153 (Springer-Verlag New York Inc., New York 1969)

[11] Satoshi Koike, The Briancon-Speder and Oka families are not bilipschits trivial, in Several topics in singularity theory, Kyoto University research information repository $\mathbf{1 3 2 8}$ (2003), 165-173. http://www.kurims.kyoto-u.ac.jp/ kyodo/kokyuroku/2003.html

[12] J.-M. Lion, J.-P. Rolin, Intégration des fonctions sous-analytiques et volumes des sous-ensembles sous-analytiques. Ann. Inst. Fourier (Grenoble) 48 (1998), 755-767.

[13] Valette, Guillaume The link of the germ of a semi-algebraic metric space. Proc. Amer. Math. Soc. 135 (2007), 3083-3090.

Departamento de Matemática, Universidade Federal do Ceará (UFC), Campus do Picici, Bloco 914, Cep. 60455-760. Fortaleza-Ce, Brasil

E-mail address: birb@ufc.br

Departamento de Matemática, Universidade Federal do Ceará (UFC), Campus do Picici, Bloco 914, Cep. 60455-760. Fortaleza-Ce, Brasil

E-mail address: alex@mat.ufc.br

Department of Mathematics, Barnard College, Columbia UniverSITY, NEW YORK, NY 10027

E-mail address: neumann@math.columbia.edu 